\title{
Thyroid Papillary Microcarcinoma Revealed by Cystic Lymph Node Metastasis
}

Tatari MM*, Anajar S, Halily S, Abada R, Rouadi S, Roubal M and Mahtar $M$

ENT Department, University Hospital Centre IBN ROCHD, Morocco

*Corresponding author: Mohamed MoutaaTatari, ENT Department, Face and Neck Surgery, Hospital August, 20'1953, University Hospital Centre IBN ROCHD, Casablanca, Morocco

Received: October 12, 2017; Accepted: October 23, 2017; Published: November 01, 2017

\begin{abstract}
Introduction: Thyroid papillary microcarcinomas revealed by cystic cervical lymph node metastasis are exceptionally observed. Cervical cystic masses are most often associated with bronchial cleft cysts.

Case Report: In this paper, we presented a 63 years old patient presenting an isolated lateral cervical cystic mass for which he was operated. Pathological examination of the surgical specimen found a lymph node metastasis of papillary carcinoma. The thyroidectomy performed afterwards found a papillary microcarcinoma that had gone unnoticed in the initial radiological examinations.

Discussion and Conclusion: Latero-cervical cysts are usually benign, especially in young subjects. But the possibility of a metastatic lesion, in particular of a thyroid carcinoma, must be raised before such situations, hence the value of a good diagnostic strategy.
\end{abstract}

Keywords: Thyroid Cancer; Papillary microcarcinoma; Lateral cervical cystic mass

\section{Introduction}

Thyroid microcarcinomas are defined according to the classification of the World Health Organization (WHO) as carcinomas of size less than or equal to $10 \mathrm{~mm}$ [1]. Because of their small size, these microcarcinomas escape cervical clinical examination and may not even be detected by imaging. Thyroid carcinomas are dominated by papillary carcinomas, which usually give metastases to the cervical lymph node. These ganglion metastases are usually solid and rarely cystic [2]. Thus, these cystic adenopathies are often interpreted as benign cystic masses [3], especially if the thyroid appears normal. The aim of this work is to report a case of papillary microcacinoma of the thyroid revealed by a cystic ganglionic metastasis initially taken for a benign primitive cervical cystic mass, and to highlight the paraclinical features that may make these lesions suspect.

\section{Clinical Case}

A 63-year-old male patient with no history of cervical irradiation or familial thyreopathy, who has had two years of progressively enlarged, and painless cervical mass with no signs of compression. The clinical examination found a right lateral cervical mass, resembling $5 \times 3 \mathrm{~cm}$, movable, without inflammatory signs (Figure 1).

No other lymph node or thyroid mass was palpated. The rest of the otolaryngological and endoscopic examination was normal. The cervical ultrasound showed two solid cystic masses with a predominant cystic component, multi-partitioned, in front of the right jugulo-carotid pedicle, the first measuring $81 \mathrm{~mm}$ long and the second 39x16mm. The thyroid gland was of normal size, with regular and homogeneous contours without a detectable nodule on this examination. Cervical CT has returned to a solid and cystic formation on the right III territory, with a fleshy portion that is enhanced intensely after injection of contrast agent. The thyroid gland appeared normal (Figure 2).
A biopsy under local anesthesia was performed with an anatomopathological result that was negative. An exploratory cervicotomy was indicated, finding a polylobed necrotic cystic mass. A monoblock excision of the entire mass was performed.
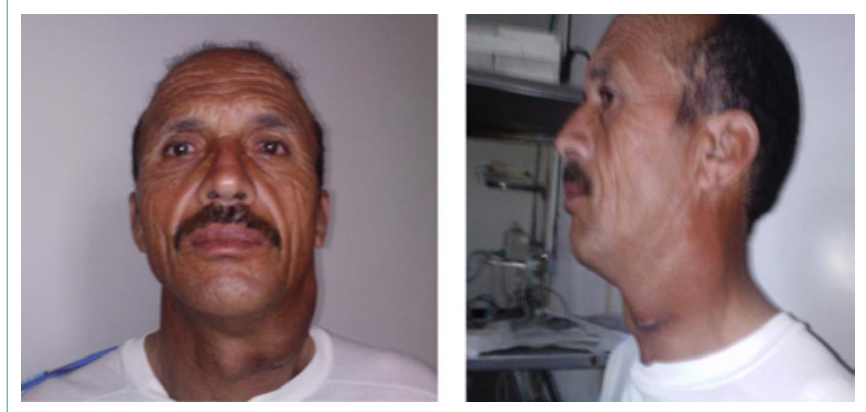

Figure 1: Clinical aspect of left lateral cervical mass.
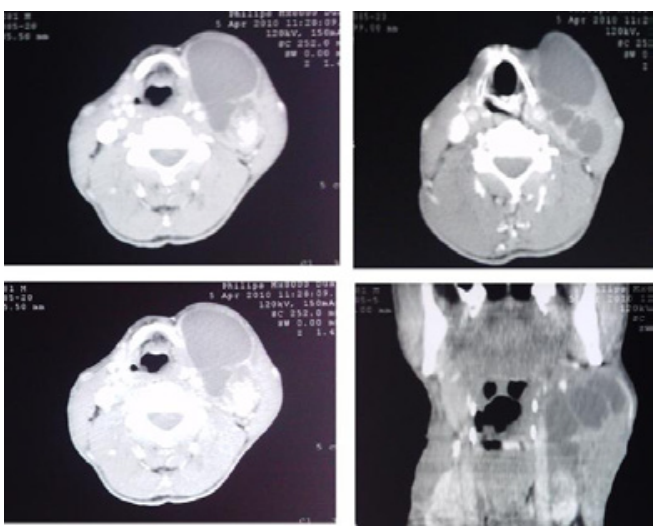

Figure 2: CT scan showing a solid cystic mass in the right III territory, with a fleshy portion that enhances intensely after injection of contrast agent.
Annals Thyroid Res - Volume 3 Issue 2 - 2017

Submit your Manuscript | www.austinpublishinggroup.com

Tatari et al. () All rights are reserved
Citation: Tatari MM, Anajar S, Halily S, Abada R, Rouadi S, Roubal M, et al. Thyroid Papillary Microcarcinoma Revealed by Cystic Lymph Node Metastasis. Annals Thyroid Res. 2017; 3(2): 120-121. 


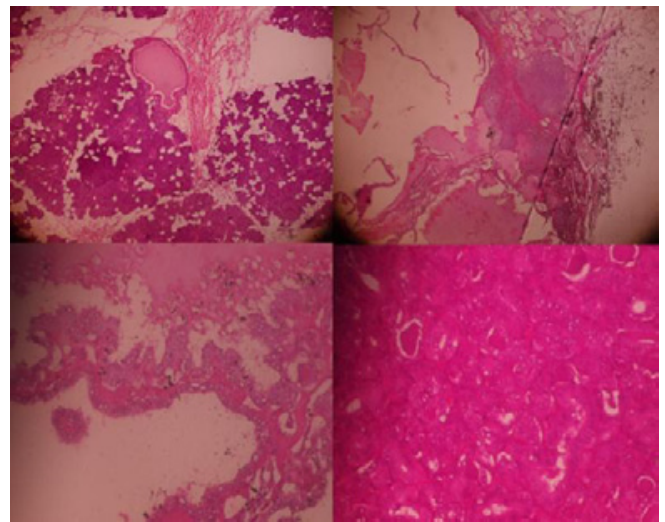

Figure 3: Papillary carcinomatous proliferation, with images of vascular emboli and necrosis foci.

The anatomopathological study of the mass demonstrated papillary carcinomatous proliferation and images of vascular emboli, foci of necrosis, as well as lymph nodes in some places evoking lymph node metastasis of papillary carcinoma (Figure 3 ).

In order to investigate the primary cancer, a second cervical ultrasound was performed postoperatively, which resumed a normalsized thyroid with regular and homogeneous contours. It also showed a right upper -hypoechogenic nodule and mixed vascularization measuring $5.5 \times 4.3 \mathrm{~mm}$. TSH us was normal at $3.24 \mu \mathrm{IU} / \mathrm{ml}(0.27$ $4.2 \mu \mathrm{IU} / \mathrm{ml}$ ). A total thyroidectomy with recurrent and right jugular lymph node dissection was performed. The anatomopathological study found a papillary micro carcinoma of $5 \mathrm{~mm}$ of major axis without lymph node involvement. The patient was subsequently referred to the Nuclear Medicine Department for further treatment.

\section{Discussion}

Latero-cervical cysts are generally benign lesions and occur mostly in young subjects. Malignant cystic lesions are less frequent and can be secondary to tumors of the oropharynx, thyroid and salivary glands [4]. The primary tumor is usually detected by a CT scan, except in the case of small thyroid tumors, especially microcarcinomas, which may go undetected [5]. The presence of an isolated cystic latero-cervical mass, as the only manifestation of occult thyroid carcinoma, is rare. To our knowledge, only about fifty similar cases are described in the literature [6,7]. The differential diagnosis of cystic latero-cervical metastases of thyroid carcinomas is mainly done with second branchial cleft cysts, which are classically located below sternocleidomastoid muscle [7].

According to some studies, CT may help differential diagnosis between benign cysts and cystic metastases. The presence of enhanced elements after injection of contrast agent within the cyst would be in favor of the metastatic nature of the thyroid carcinoma [8], as was the case of this patient.. Cervical ultrasound may also provide some evidence for the metastatic origin of cervical cystic masses, such as the presence of thick cystic walls, nodules and intracystic walls [9], as was the case with our patient who presented a multi-partitioned cyst with initial cervical ultrasound. The FNAC (Fine Needle Aspiration Cytology) allows the diagnosis of the thyroid lesion type with a diagnostic accuracy of $92-95 \%$. It has two important limitations: inadequacy of the samples for cytology and follicular proliferation [11]. The cytopunction allows a histological orientation of the cervical masses, especially in case of solid adenopathies. However, it does not have the same efficacy in cystic masses [10], and may not find malignant cells in real cystic lymph node metastases with a false negative rate of $50 \%$ [7]. The determination of thyroglobulin in the aspirated liquid of the cyst associated with cytology can improve the preoperative diagnosis of cystic metastases of thyroid carcinomas, thus avoiding the need for further complementary examinations. Indeed, the high concentration of thyroglobulin in the aspirated fluid would be specific to papillary carcinoma metastases [7]. Treatment of such lesions consists of total thyroidectomy with a suitable lymph node dissection for the stage of the tumor. Radioiodine therapy is recommended for patients with lymph node metastasis, even for the microcarcinomas according to the latest recommendations of the American Thyroid Association, which classify these patients as being at intermediate risk [6].

\section{Conclusion}

Latero-cervical cysts are usually benign, especially in young subjects. But the possibility of a metastatic lesion, in particular of a thyroid carcinoma, must be raised in such situations hence the value of a good diagnostic strategy based on the clinical, radiological and cytological description of adenopathies.

\section{References}

1. Hedinger C, Williams ED, Sobin LH. The WHO histological classification of thyroid tumors: A commentary on the second edition. Cancer. 1989; 63: 908-911.

2. Miseikyte-Kaubriene E, Trakymas M, Ulys A. Cystic lymph node metastasis in papillary thyroid carcinoma. Medicina (Kaunas). 2008; 44: 455-459.

3. Ahuja A, Ng CF, King W, Metreweli C. Solitary cystic nodal metastasis from occult papillary carcinoma of the thyroid mimicking a branchial cyst: a potential pitfall. ClinRadiol. 1998; 53: 61-63.

4. Compagno J, Hyama VJ, Safarian M. Does branchiogenic carcinoma really exist? Arch Pathol Lab Med. 1976; 100: 311-316.

5. Inagaki Y, Sakamoto K, Inoue Y, Imanishi Y, Tomita T, Shinden S, et al Thyroglobulin concentration measurement in fine-needle aspiration fluid from cystic cervical lymph node metastases of papillary thyroid carcinoma. Nihon Jibiinkoka Gakkai Kaiho. 2011; 114: 912-916.

6. Haugen BR Md, Alexander EK, Bible KC, Doherty GM, Mandel SJ, Nikiforov YE, et al. 2015 American Thyroid Association Management Guidelines for Adult Patients with Thyroid Nodules and Differentiated Thyroid Cancer. Thyroid. 2015.

7. Ozdamar OI, Acar GO, Kafkasli C, Tayyar Kalcioglu M, Tulay Zenginkinet, Gonca Tamer H. Papillary Thyroid Microcarcinoma with a Large Cystic Dilated Lymph Node Metastasis to the Neck Mimicking a Branchial Cleft Cyst: A Potential Pitfall. Case Rep Otolaryngol. 2015; 796358.

8. Nakagawa T, Takashima T, Tomiyama K. Differential diagnosis of a latera cervical cyst and solitary cystic lymph node metastasis of occult thyroid papillary carcinoma. J Laryngol Otol. 2001; 115: 240-242.

9. Wunderbaldinger P, Harisinghani MG, Hahn PF, Daniels GH, Turetschek $\mathrm{K}$, Simeone J, et al. Cystic lymph node metastases in papillary thyroid carcinoma. AJR Am J Roentgenol. 2002; 178: 693-697.

10. Verge J, Guixá J, Alejo M, Basas C, Quer X, De Castro J, et al. Cervica cystic lymph node metastasis as first manifestation of occult papillary thyroid carcinoma: report of seven cases. Head Neck. 1999; 21: 370-374.

11. Berker D, Aydin Y, Ustun I, Gul K, Tutuncu Y, Işik S, et al. The value of fineneedle aspiration biopsy in subcentimeter thyroid nodules. Thyroid. 2008; 18: $603-608$. 